\title{
Two-Site Optical Observation and Initial Orbit Determination for Geostationary Earth Orbit Satellites
}

\author{
Jin Choi ${ }^{\dagger}$, Young-Jun Choi, Hong-Suh Yim, Jung Hyun Jo, and Wonyong Han
}

Korea Astronomy and Space Science Institute, Daejeon 305-348, Korea

Optical observation system provides angle-only measurement for orbit determination of space object. Range measurement can be directly acquired using laser ranging or tone ranging system. Initial orbit determination (IOD) by using angle-only data set shows discrepancy according to the measurement time interval. To solve this problem, range measurement data should be added for IOD. In this study, two-site optical observation was used to derive the range information. We have observed nine geostationary earth orbit satellites by using two-site optical observation system. The determination result of the range shows the accuracy over 99.5\% compared to the results from the satellite tool kit simulation. And we confirmed that the orbit determination by the Herrick-Gibbs method with the range information obtained from the two-site observation is more accurate than the orbit determination by Gauss method with the one-site observation. For more accurate two-site optical observation, a baseline should satisfy an optimal condition of length and more precise observation system needed.

Keywords: two-site observation, initial orbit determination

\section{INTRODUCTION}

In general, space surveillance system consists of optical, radar and laser systems. Radar and laser systems observe satellites and debris from low earth orbit (LEO) to medium earth orbit (MEO). On the other hand, optical system chiefly observes satellites and space debris on geostationary earth orbit (GEO) (Lee et al. 2004). Also the optical system is restrictively used for observation of LEO or MEO satellites (Lee 2003).

The single site of optical observation can provide not the range measurement but the angle-only measurement. The range is calculated with time span for observations and with angle data. The velocity of satellite is also calculated with the range. This process, called initial orbit determination (IOD), needs at least three times of independent observations, because the range and velocity vectors consist of 3 components in coordinate system respectively (Montenbruck \& Gill 2000).

IOD should be improved by the process such as differential correction and follow-up observations, because the orbital elements of IOD are not accurate to keep tracking the satellite, due to a large observational error or a short time span (Musci et al. 2004).

IOD result of optical observation can be obtained from the total time span over 3 times of observations using an optical telescope. The accuracy of IOD depends on orbit determination method and time span between exposures. For example, the accuracy of result for IOD with Gauss method is various with time span (Kim et al. 1988). Gauss method implicitly uses range information of the middle over the observational time span, to solve the equation of motion. This indirect information of the range, but, is affected by the time span or the accuracy of observations.

However, the range information can be calculated by (c) This is an Open Access article distributed under the terms of the Creative Commons Attribution Non-Commercial License (http://creativecommons.org/licenses/by-nc/3.0/) which permits unrestricted non-commercial use, distribution, and reproduction in any medium, provided the original work is properly cited.
Received Jul 14, 2010 Revised Sep 30, 2010 Accepted Nov 15, 2010 ${ }^{\dagger}$ Corresponding Author

E-mail: rutcome@kasi.re.kr

Tel: +82-42-865-2080 Fax: +82-42-861-5610 
trigonometric method using optical telescopes. The annual parallax is good example for calculation of range from the earth to nearby star. The annual parallax uses the revolution way of the earth as baseline because the star is relatively stationary and the range to the star is very far with respect to the baseline. In the case of artificial satellite, the baseline can be limited to earth's radius. Trigonometric parallax using two different telescopes at the same time needs sufficient time synchronization and the observational accuracy.

In general, simultaneous optical observation has not been used main option for GEO satellite tracking. Porfilio et al. (2006) pointed out two drawbacks related the simultaneous observation which the weather and observatory condition and the altitude limitation for the satellite orbit. The joint campaign for two-site orbit determination was implemented in September 2003. The observation system, the Group of Astrodynamics of the University of Rome "La Sapienza" (GAUSS), consisted of two optical telescopes with $1,000 \mathrm{~km}$ of direct distance, between Collepardo and Mallorca. The results of the two-site orbit determination were outstanding when compared to those of the one-site orbit determination (Porfilio et al. 2006). Another attempt for GEO satellite using CASTOR II and SMART-Scope with $30 \mathrm{~km}$ of the baseline for endto-end of Ottawa, resulted in not enough accuracy due to the short baseline (Earl 2005).

In this paper, we observed GEO satellites with two optical observation systems at Daejeon Observatory in the Korean Astronomy and Space science Institute (KASI) and at Siding Springs Observatory (SSO) in Australia. We calculated the range for each satellite and implemented

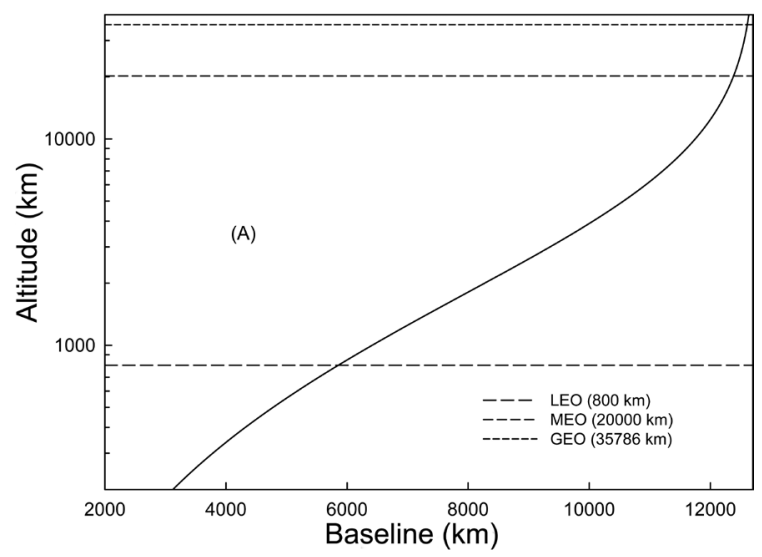

Fig. 1. Baseline upper limit for two-site optical observation due to the difference of the visibilities of two sites. Selected region (A) indicates that satellite can be observable from both telescopes. Dashed lines indicate typical LEO, MEO and GEO altitude respectively. LEO: low earth orbit, MEO: medium earth orbit, GEO: geostationary earth orbit. the IOD process. Then, the results were compared with the results for one site orbit determination through the Gauss method.

This study is not for a regular GEO satellites tracking but for the space surveillance. Generally, techniques like a tone ranging and an angle tracking are used for orbit determination for GEO satellites tracking (Soop 1994). But in case of space surveillance the target for tracking include space objects not only able to communicate but also uncontrollable. Therefore the optical observation is still useful to surveillant for various space objects. And also it is impossible to know the orbital elements for some space objects. In this case, IOD is general method to know the initial state of the space object, especially with optical system.

\section{CONDITIONS FOR TWO-SITE OPTICAL OB- SERVATION}

The two-site optical observation for an artificial satellite has definite limit conditions like baseline, time synchronization and weather condition. Basically, the angle resolution of the optical observation is higher with the longer baseline in case of two-site observation. However, in case of the LEO satellite, the lower altitude limitation occurs due to the baseline length and the curvature of the Earth. In case of GEO satellite, mainly the length of the baseline affects the accuracy of the observation.

Fig. 1 shows the theoretical limit condition that satellite in a typical altitude can be observable from both telescopes with a given baseline. For the typical LEO satellite,

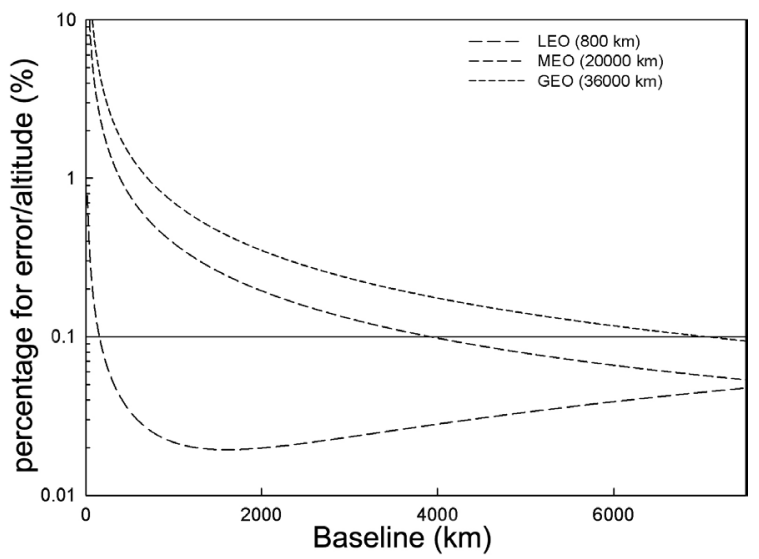

Fig. 2. Relative error with respect to altitude for the baseline. Two-site observation of LEO satellites has the minimum error when the baseline is about 1,600 km. LEO: low earth orbit, MEO: medium earth orbit, GEO: geostationary earth orbit. 
we selected the altitude of $800 \mathrm{~km}$ through the stats from two line elements (TLE) data. LEO satellites with the altitude between 600 and $800 \mathrm{~km}$ numbered more than 50 percent of all LEO satellites.

We assumed, in this calculation, only the mean Earth radius 6,378.1366 km (The Astronomical Almanac 2010) and an elevation of 0 degree. Although the Earth is not circular flattened, the telescopes can be located at any place on the surface of the Earth.

The theoretical limit condition for two-site observation means possible maximum baseline against the altitude of satellite. In the case of LEO satellite, maximum baseline is about $5,800 \mathrm{~km}$, which means that satellites lower than $800 \mathrm{~km}$ can't be observed at the same time from both sites. On the other hand, the theoretical limit condition is more than $12,000 \mathrm{~km}$ for MEO and GEO satellites. Therefore the maxi-mum baseline for satellites with altitude higher than MEO is almost unlimited on the surface of the earth.

Fig. 2 shows the relative error with respect to altitude when we assume an total error of 20 arcseconds for each optical observation system (see next section). This 20 arcseconds error includes the optical system error, charge-coupled device (CCD) pixel size error, time synchronization, atmospheric refraction.

LEO satellite can be observed theoretically under relative error of one percent of the altitude of satellite at the time for all baselines. But the observation with the baseline longer than $160 \mathrm{~km}$ can give the result under 0.1 percent of the relative error. The relative error for LEO satellites shows the minimum value at the baseline near 1,600 $\mathrm{km}$. Satellites on MEO and GEO need the baseline longer than $4,000 \mathrm{~km}$ and $7,000 \mathrm{~km}$, respectively, for smaller relative error then 0.1 percent. But the relative error for satellites in MEO and GEO decreases continuously as the baseline goes long.

GAUSS team pointed out the issues for the optimal baseline for two-site observation. The best accuracy in determining the target position is achieved when the observing directions intersect with an angle of 90 degrees (Porfilio et al. 2006). We confirmed that the minimum error occurs at the $1,600 \mathrm{~km}$ baseline length in case of the $800 \mathrm{~km}$ altitude LEO satellite. It coincided exactly with the GAUSS team's result. Of course, the MEO and GEO satellite's optimal baseline is about 40,000 and $85,000 \mathrm{~km}$ respectively, which is not realistic for the surface of the Earth.

Therefore, for LEO satellite, the optimal baseline is between $160 \mathrm{~km}$ and $1,600 \mathrm{~km}$. The optimal baselines for MEO and GEO satellite should be longer than 4,000 km and 7,000 km, respectively.

\section{TWO-SITE OPTICAL OBSERVATION}

Optical observatories for two-site observation have the robotic telescope. Both telescopes are established for the research of near earth object, operated by Near Earth Space Survey (NESS) team. The first one, named Daedeok Observatory (DO), is located at KASI, Republic of Korea, on latitude of $36^{\circ} 23^{\prime} 53^{\prime \prime} \mathrm{N}$, longitude of $127^{\circ} 22^{\prime} 31^{\prime \prime} \mathrm{E}$ and altitude of $100 \mathrm{~m}$. The other one is located at SSO, Australia on latitude of $31^{\circ} 16^{\prime} 25^{\prime \prime} \mathrm{S}$, longitude of $149^{\circ} 3^{\prime} 51^{\prime \prime} \mathrm{E}$ and altitude of 1,165 m. Fig. 3 shows the location of each sites. The baseline between two sites is about 7,368 km.

Fig. 4 shows the telescopes used for two-site observation. DO telescope (left) has a primary mirror of $0.6 \mathrm{~m}$ diameter with, f-ratio of 2.92 , equipped with at the prime focus, $2 \mathrm{k} \times 2 \mathrm{k}$ Apogee U10 CCD having field of view (FOV) of 0.89 degrees and pixel scale of 1.83 arcsecond / pixel. SSO telescope (right) has a primary mirror of 0.5 $\mathrm{m}$ diameter with f-ratio of 1.89 , equipped with $2 \mathrm{k} \times 2 \mathrm{k}$ Apogee primary mirror of U10 CCD, having FOV of 1.89 degrees and pixel scale of 3.02 arcsecond / pixel. And both telescopes have the open fork mount and a limit on lowest elevation of 30 degrees due to mechanical limit.

GEO satellites are the best candidate to meet the condition of very long baseline and the limitation of each telescope. Almost 76 percent GEO satellites in the public data of TLE have an inclination under 2 degrees. We select 11 GEO satellites which have small inclination and the opposite one among the observable candidate from both sides at the same time.

The two-site observations were tried during two nights from 21 to 22 January, 2010. Observations were performed with schedule mode and the settings of the telescopes were sidereal tracking mode. We observed a satellite for 30 minutes with exposure time of 5 seconds and with interval of 60 seconds. As a result, we failed the observation for JCSAT-4A and INTELSAT 8 due to weak reflection light. Since the time synchronization between the two systems is important, our observation systems were synchronized in 1 second with global positioning system signal. The error of the time synchronization came from the system delay and the truncation error by CCD header.

Observed satellite is seen as a streak with respect to field stars. On average, streaks of this observation have about 3 pixels of width, equivalent to 6-10 arcseconds. And also there are the control system error, time syn- 


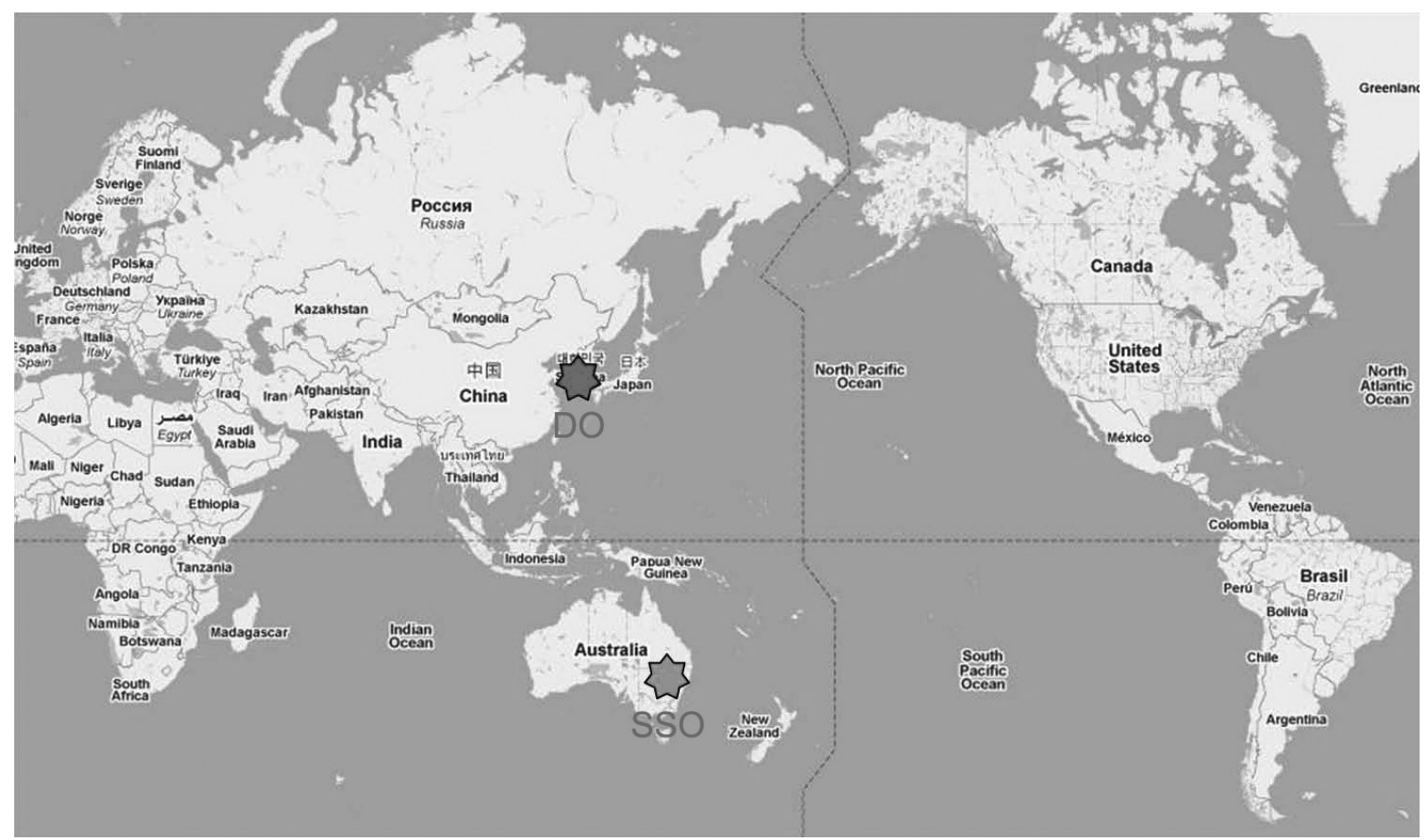

Fig. 3. Optical observatories for two-site observation are located at Korean Astronomy and Space science Institute (KASI), Republic of Korea and at Siding Springs Observatory (SSO), Australia.
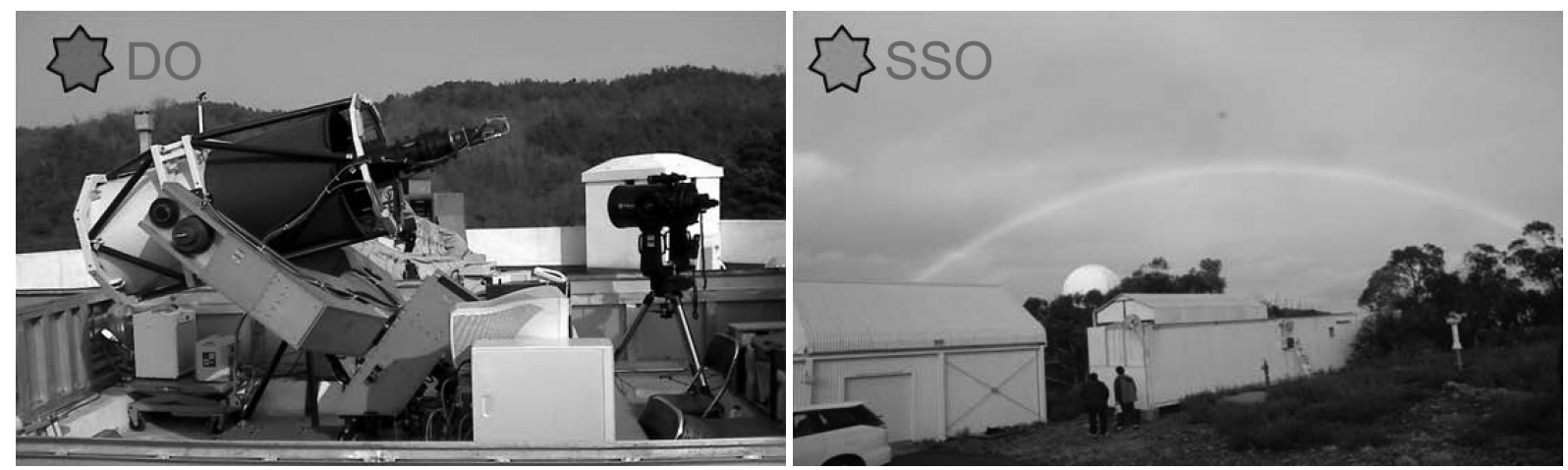

Fig. 4. Daedeok Observatory (DO) telescope (left) and Siding Springs Observatory (SSO) telescope (right) were used for two-site observation.

chronization, and atmospheric refraction. Therefore, the maximum error of observed position for satellite was about 20 arcseconds.

The observation system also has positioning error and the distortion due to the optical system structure. We corrected the error with the world coordinate system (WCS) solution (Greisen \& Calabretta 2002) and the distortion correction.

\section{DETERMINING THE RANGE WITH OPTICAL OBSERVATION}

Observational error is in inverse proportion to trigonometric parallax. As described in previous section, the error of the observation system is about 20 arcseconds on average.

We applied a least square fit to the position data over 30 minutes observations to obtain a representative value at given time and Fig. 5 shows difference of observed value and representative value. Fig. 6 shows the trigonometric parallax of GEO satellites with the baseline $7,368 \mathrm{~km}$ between DO and SSO. Observational error is negligible because the parallax is longer than 11 degrees for our targets of GEO satellites. 


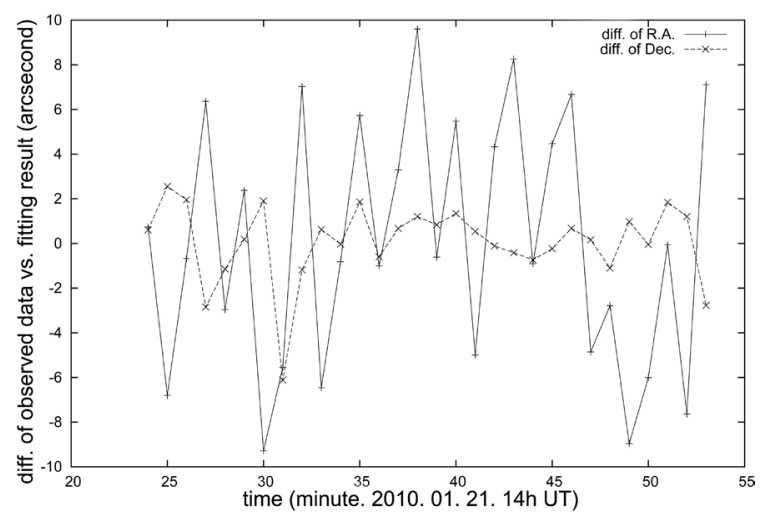

Fig. 5. Difference between the position observed and the data obtained by least square method for APSTAR 1, shows less than 10 arcseconds in right ascension and declination.

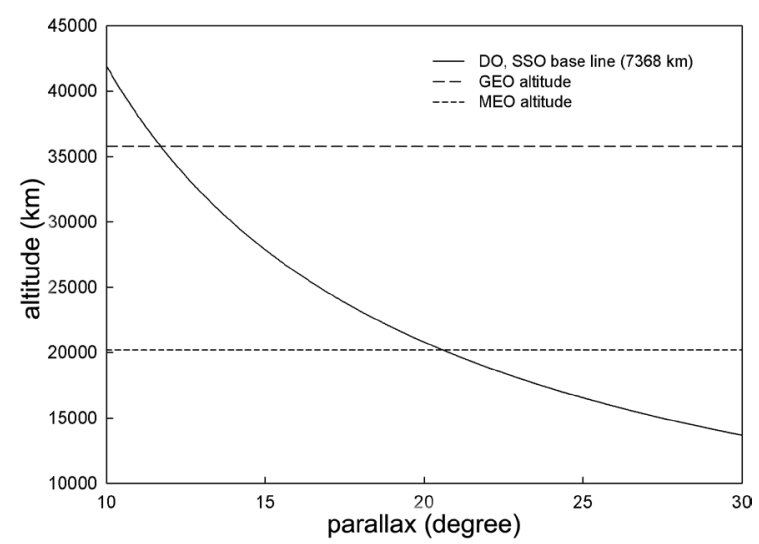

Fig. 6. Parallax for GEO satellite is about 11 degrees, while MEO satellite can be observed with the trigonometric parallax almost 20 degrees. DO: Daedeok Observatory, SSO: Siding Springs Observatory, GEO: geostationary earth orbit, MEO: medium earth orbit.

Table 1. The range to satellite from DO and SSO.

\begin{tabular}{llllll}
\hline \multirow{2}{*}{ Satellite name } & \multicolumn{2}{c}{ DO } & & \multicolumn{2}{c}{ SSO } \\
\cline { 2 - 3 } \cline { 5 - 6 } & Range $(\mathbf{k m})$ & Accuracy (\%) & & Range $(\mathbf{k m})$ & Accuracy (\%) \\
\hline APSTAR 1 & $37,134.12$ & 99.9873 & & $37,169.52$ & 99.99431 \\
SUPERBIRD-B2 & $38,245.91$ & 99.98583 & & $37,030.5$ & 99.97671 \\
AGILA 2 & $37,312.64$ & 99.71903 & & $36,669.16$ & 99.3618 \\
BIEDOU 1A & $37,327.14$ & 99.708 & & $36,988.68$ & 99.83456 \\
GARUDA 1 & $37,250.95$ & 99.889 & & $37,468.08$ & 99.2025 \\
JCSAT-2A & $37,835.54$ & 99.70612 & & $36,893.27$ & 99.88827 \\
JCSAT-R & $37,249.8$ & 99.94645 & & 37,850 & 99.93029 \\
N-STAR C & $35,803.22$ & 96.13806 & & $35,787.7$ & 96.24314 \\
SUPERBIRD-C & $37,376.28$ & 98.66912 & & $36,970.84$ & 99.92451 \\
\hline
\end{tabular}

DO: Daedeok Observatory, SSO: Siding Springs Observatory.

Table 1 shows the result of determining the range we determined of 9 GEO satellites. For most of the cases, the determined accuracy is over $99.5 \%$ except for N-STAR C. Since the N-STAR C don't have enough brightness for determining the end-point of the streak, the position error

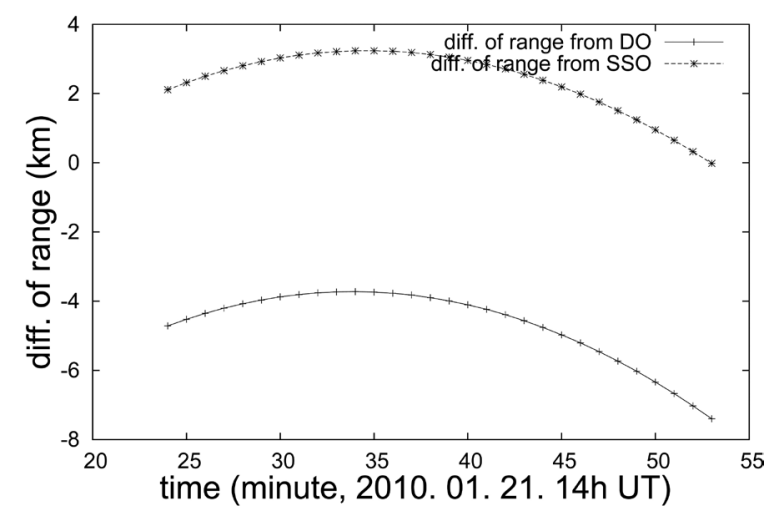

Fig. 7. Difference between the range from satellite tool kit and the range calculated by the trigonometric method for APSTAR 1. DO: Daedeok Observatory, SSO: Siding Springs Observatory.

is unexpectedly larger than the others.

The result also consists with previous study for the accuracy of two-site observation. The aforementioned two campaigns showed that the accuracy was too bad to determine the range with a short baseline of two telescopes. GAUSS team used the baseline about 1,000 km and the other team used the baseline of only $30 \mathrm{~km}$ (Earl 2005, Porfilio et al. 2006).

Fig. 7 shows total average of the difference of the range for the calculation with satellite tool kit (STK) using TLE and the result by the trigonometric method.

\section{IOD WITH RANGE AND ANGULAR DATA}

As mentioned earlier, IOD result is very important to keep tracking the satellite with the optical observation system. In the case of one-site observation, the range to the satellite determines indirectly.

Gauss method is a typical and famous method for general one-site observation using optical telescope (Kim et al. 1988, Vallado \& McClain 2001). The range at middle time is determined by solving a polynomial equation of 8th degree. This process needs the assumption that the motion of satellite can be described as two-body motion and time span, and that 3 sets of position data consist of topocentric right ascension and declination, or azimuth and elevation with time. Accuracy of the solution of the polynomial equation is affected by the error of the position and time span (Escobal 1975).

The IOD method using range and angular data is Herrick-Gibbs method. Herrick-Gibbs method is used for determining the velocity at the second point of observations, while Gauss method is used for determining the range at the second (Escobal 1975). 

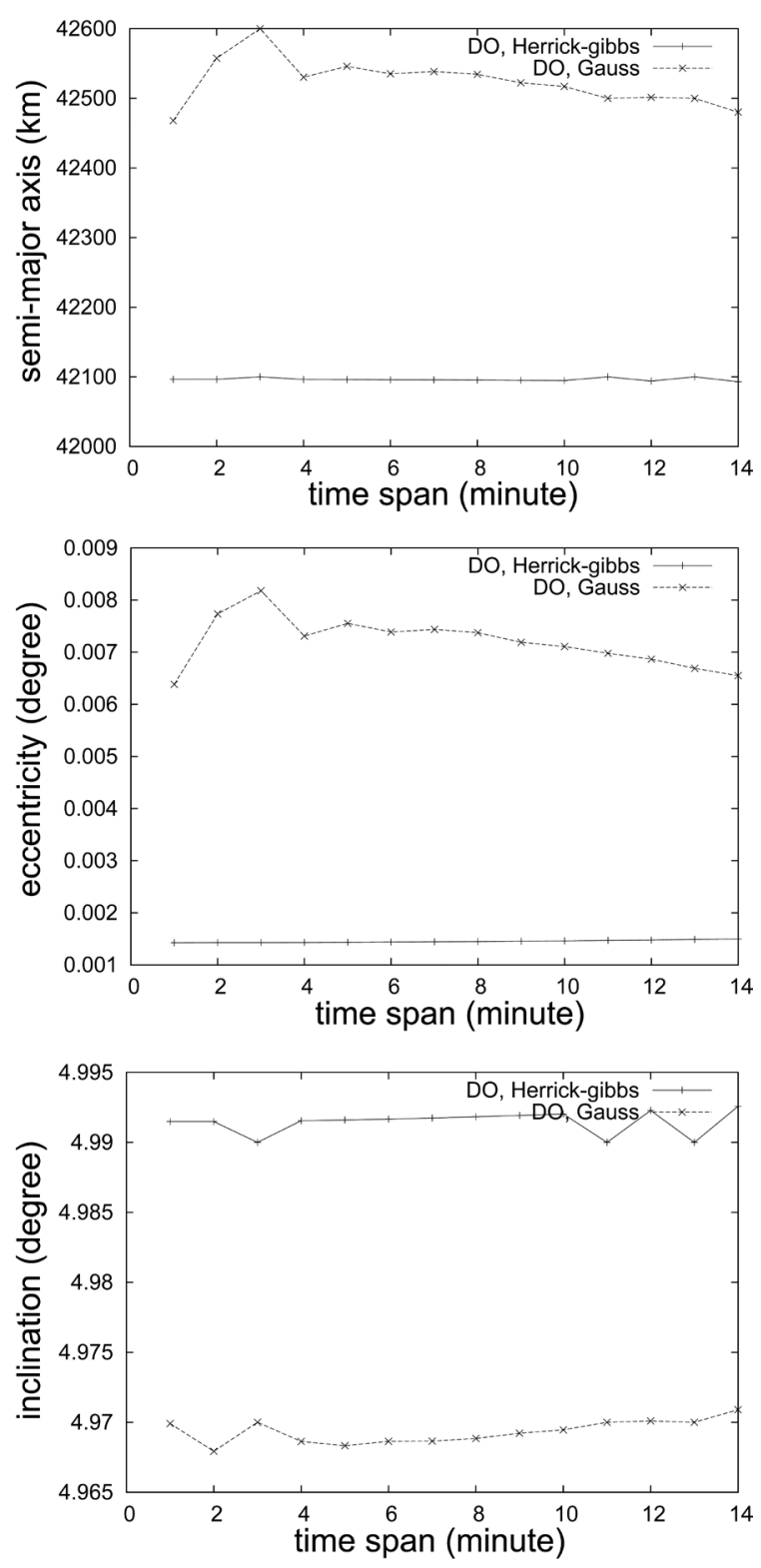

Fig. 8. The initial orbit determination result calculated for APSTAR 1 from Gauss method and Herrick-Gibbs method for semi-major axis, eccentricity and inclination. The result using the Gauss method is irregular with time-span.
Fig. 8 shows the result calculated from Gauss method and Herrick-Gibbs method. The orbital elements using Gauss method show irregular pattern with time span while those using Herrick-Gibbs method are steady with time span.

Table 2 shows the result using Herrick-Gibbs method and the Gauss method at DO. The time span was selected to make the best results for each satellite. The best time span is 10 minutes which are also the maximum value as possible for the most satellite. Herrick-Gibbs method showed the best result in semi-major axis for all satellites, and better results in eccentricity and inclination than by Gauss method.

\section{IMPROVEMENT OF THE IOD RESULT WITH THE DIFFERENTIAL CORRECTION}

Generally, because the IOD result is not accurate enough to use for tracking the satellite with long period or for using telescope with small FOV. It requires an improvement with extra data. GAUSS team says that there are two ways to accomplish a complete orbit determination: follow-up observations or two-site surveys (Porfilio et al. 2006).

Even though we tried two-site surveys, the result can be improved with the differential correction. We used it as only a statistical method which is not including any dynamics.

Fig. 9 shows the difference of ephemeris between improved by differential correction and obtained from North American Aerospace Defense Command (NORAD) TLE. The difference of Right ascension and declination using Herrick-Gibbs method improved by differential correction, is more steady than those using Gauss method. The difference between them is smaller than 20 arcseconds which is equivalent to the observational error.

Table 2. The result using the Herrick-Gibbs method and the Gauss method at DO.

\begin{tabular}{|c|c|c|c|c|c|c|c|c|c|}
\hline \multirow{2}{*}{ Satellite } & \multicolumn{3}{|c|}{ TLE } & \multicolumn{3}{|c|}{ Herrick-Gibbs } & \multicolumn{3}{|c|}{ Gauss } \\
\hline & $\mathrm{a}(\mathbf{k m})$ & e & i (degree) & $\mathrm{a}(\mathbf{k m})$ & e & i (degree) & $\mathrm{a}(\mathbf{k m})$ & e & i (degree) \\
\hline APSTAR 1 & $42,088.76$ & 0.0001160 & 4.9191 & $42,094.71$ & 0.001461 & 4.992031 & $42,517.1$ & 0.007108 & 4.969464 \\
\hline SUPERBIRD-B2 & $42,087.69$ & 0.0001971 & 0.0364 & $42,039.82$ & 0.002535 & 0.015084 & $42,219.34$ & 0.000665 & 0.016873 \\
\hline AGILA 2 & $42,164.4$ & 0.0005104 & 0.066 & $41,212.25$ & 0.017782 & 0.028865 & $42,110.97$ & 0.001772 & 0.026877 \\
\hline BIEDOU 1A & $42,164.8$ & 0.0003364 & 1.0905 & $42,147.56$ & 0.000535 & 1.163593 & $30,900.86$ & 0.243591 & 1.476048 \\
\hline GARUDA 1 & 42,165 & 0.0002767 & 1.3395 & $42,215.18$ & 0.001137 & 1.299442 & $47,466.05$ & 0.086871 & 1.348257 \\
\hline JCSAT-2A & $42,164.6$ & 0.0003831 & 0.0424 & $42,232.98$ & 0.0024 & 2.133657 & $40,476.37$ & 0.031094 & 0.080719 \\
\hline JCSAT-R & $42,164.3$ & 0.0002657 & 2.0532 & $42,232.98$ & 0.0024 & 2.133657 & $43,698.89$ & 0.0257 & 2.089492 \\
\hline N-STAR C & $42,164.9$ & 0.000272 & 0.0559 & $41,130.23$ & 0.288042 & 1.780609 & $36,227.42$ & 0.117919 & 0.223249 \\
\hline SUPERBIRD-C & 42,165 & 0.0002887 & 1.1479 & $42,130.4$ & 0.00068 & 1.229468 & $33,893.32$ & 0.16949 & 1.556578 \\
\hline
\end{tabular}

DO: Daedeok Observatory. 

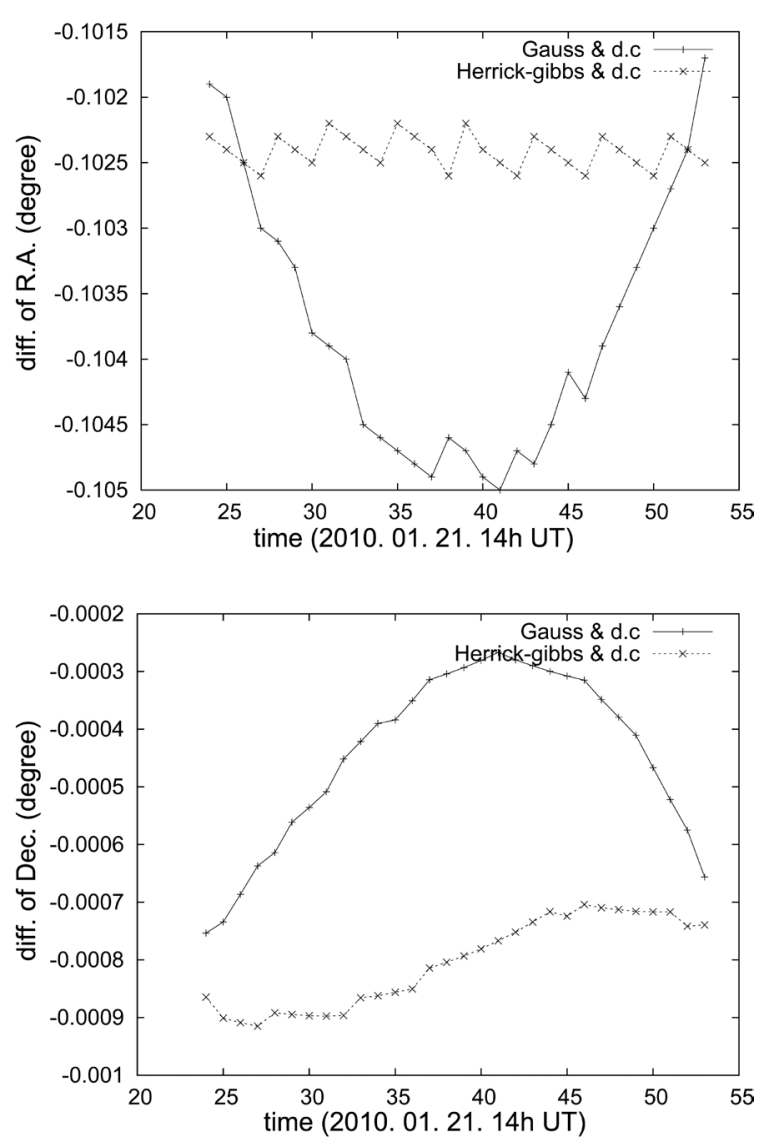

Fig. 9. Each initial orbit determination results was improved by the differential correction. It was converted formation of TLE type. Ephemeris of the propagation with two line element (TLE) from North American Aerospace Defense Command (NORAD) used as a true value for the test.

\section{CONCLUSIONS}

The optimal length of baseline should be considered for two-site observation for the range information. If the error of the optical observation system is overall 20 arcseconds, the optimal length of baseline for LEO satellite observation is from 160 to $1,600 \mathrm{~km}$. The optimal baselines for MEO and GEO observation are over 4,000 and $7,000 \mathrm{~km}$ respectively.

The two-site observation is useful for the IOD. The range to satellite is calculated directly and it shows more accurate (over 99.5\%) results with the optimal baseline. Herrick-Gibbs method using the range information from two-site observation gave a stable results for various time spans. We also confirmed that differential correc- tion presents more accurate result with Herrick-Gibbs method. The difference between the value by calculation with TLE data and the value obtained from Herrick-Gibbs method followed by differential correction, shows less 20 arcseconds which is equivalent to the observational error that we assumed at the beginning.

The two-site observation is obviously useful compared with the one-site observation. To determine an accurate range, however, we should take into account the optimal length of baseline for the altitude of satellite, the astrometric error originated from the telescope and detector system, and the synchronization of the time observed from both observatories.

Two-site observation system with a small observational error and an optimal baseline can be a good solution for optical surveillance system of space objects.

\section{REFERENCES}

Earl, M. A. 2005, JRASC, 99, 50

Escobal, P. R. 1975, Methods of Orbit Determination (New York: John Wiley \& Sons), pp.250-251, pp.304-309

Greisen, E. W. \& Calabretta, M. R. 2002, A\&A, 395, 1061, doi: 10.1051/0004-6361:20021326

Kim, C. H., Shin, J. S., Park, P. H., Kim, D. H., Lee, B. S., Jo, J. H., Lee, J. S., Park, S. Y., \& Choi, G. H. 1988, JASS, 5, 1

Lee, D. J. 2003, PhD Thesis, Kyung-Hee University

Lee, W. K., Lim, H. C., Park, P. H., Youn, J. H., Yim, H. S., \& Moon, H. K. 2004, JASS, 21, 3

Montenbruck, O. \& Gill, E. 2000, Satellite Orbits: Models, Methods, and Applications (Heidelberg: Springer), pp.43-44

Musci, R., Schildknecht, T., \& Ploner, M. 2004, AdSpR, 34, 912, doi: 10.1016/j.asr.2003.01.019

Porfilio, M., Piergentili, F., \& Graziani, F. 2006, AdSpR, 38, 2084, doi: 10.1016/j.asr.2006.06.004

Soop, E. M. 1994, Handbook of Geostationary Orbits (Boston: Kluwer Academic Publisher), pp.227-235

The Astronomical Almanac. 2010, 2010 Selected Astronomical Constants [Electronic Document] Available from: http://asa.usno.navy.mil/SecK/2010/Astronomical_ Constants_2010.pdf

Vallado, D. A. \& McClain, W. D. 2001, Fundamentals of Astrodynamics and Applications, 2nd ed. (Boston: Kluwer Academic Publisher), pp.411-412 